\title{
Theoretical implications of the stress and magnetic field splitting of the GR1 line in diamond
}

\author{
J E Lowther and A M Stoneham $\uparrow$ \\ Department of Physics, University of the Witwatersrand, Johannesburg, South Africa
}

Received 24 November 1977

\begin{abstract}
The $g$ value of the excited state associated with the GR 1 line is analysed in terms of the Coulson-Kearsley defect-molecule model for the vacancy in diamond. It is shown that the experimentally observed $g$ value is entirely consistent with the expected ${ }^{1} T_{2}$ level of the model provided quenching by the dynamic Jahn-Teller effect is considered. This quenching is estimated from stress splitting data for the GR1 line. The result supports strongly the view that the GR1 centre is the neutral vacancy.
\end{abstract}

\section{Introduction}

Recently Douglas and Runciman (1977) obtained a value for the $g$-value of the excited state associated with the GR1 line observed in diamond. The low value obtained for this excited state $\left(1.68 \times 10^{-2}\right)$ indicates that the state has zero spin; the absence of temperature dependence further shows that the ground state has spin zero too. These results suggest the excited state is the ${ }^{1} \mathrm{~T}_{2}$ state given by the Coulson-Kearsley 'defect molecule' model of the neutral vacancy (see Coulson and Kearsley 1957, Coulson and Larkins 1971). On the basis of this interpretation the $g$-value is associated with a transferred orbital moment involving the four dangling bonds surrounding the vacancy. It is evident from the stress splitting of the GR1 doublet (Clark and Walker 1973 and Davies and Penchina 1974) that there is a strong dynamic Jahn-Teller effect associated with the GR1 centre. This is in accord with earlier predictions (Lannoo and Stoneham 1968) and subsequent theoretical analyses of the stress splitting and optical properties of this system (Stoneham 1975, 1977 and Lowther 1975, 1976). Accordingly, not only is the observed $g$ value associated with a delocalised magnetic moment, but also the Zeeman operator in the ${ }^{1} \mathrm{~T}_{2}$ state is quenched because of the dynamic Jahn-Teller interaction. The magnitude of this Jahn-Teller quenching can be evaluated from the reported stress splittings of the GR1 transitions or from theoretical studies of the elastic properties of the centre (Larkins and Stoneham 1971). In this paper we calculate the $g$ value of ${ }^{1} \mathrm{~T}_{2}$, and show the result compares very favourably with the value reported by Douglas and Runciman (1977).

† Permanent address: Theoretical Physics Division, AERE Harwell, Didcot, Oxon, UK. 


\section{Theory}

Coulson and Kearsley (1957) have described the various one-electron configurations of the neutral vacancy that can give rise to a many-electron state ${ }^{1} \mathrm{~T}_{2}$. Following an earlier approach (Lowther 1975) we write the state in terms of the contributions of three important configurations:

$$
{ }^{1} \mathrm{~T}_{2}=\alpha\left|s^{2} t^{2} ;{ }^{1} \mathrm{~T}_{2}\right\rangle+\beta\left|s t^{3} ;{ }^{1} \mathrm{~T}_{2}\right\rangle+\gamma\left|t^{4} ;{ }^{1} \mathrm{~T}_{2}\right\rangle
$$

where the eigenvectors may be obtained from the matrices (see Yamaguchi 1962) using the integrals given by Coulson and Larkins (1971). Similarly other important manyelectron states may be written:

$$
\begin{aligned}
& { }^{1} \mathrm{E}=P\left|s^{2} t^{2} ;{ }^{1} \mathrm{E}\right\rangle+Q\left|s t^{3} ;{ }^{1} \mathrm{E}\right\rangle+R\left|t^{4} ;{ }^{1} \mathrm{E}\right\rangle \\
& { }^{3} \mathrm{~T}_{1}=L\left|s^{2} t^{2} ;{ }^{3} T_{1}\right\rangle+M\left|s t^{3} ;{ }^{3} \mathrm{~T}_{1}\right\rangle+N\left|t^{4} ;{ }^{3} \mathrm{~T}_{1}\right\rangle .
\end{aligned}
$$

Stress measurements have been performed on the ground state of the GR1 system and, taking ${ }^{1} \mathrm{E}$ as the ground state of the GR1 transition, we obtain the following values for the stress parameters:

$$
\begin{aligned}
& D=\left\langle\theta\left|C_{E \epsilon}\right| \epsilon\right\rangle=-\frac{2}{\sqrt{3}}\langle\epsilon|C| \epsilon\rangle C\left({ }^{1} E\right) q \\
& B=\frac{2}{\sqrt{3}}\left\langle T_{x}\left|C_{E \epsilon}\right| T_{x}\right\rangle=-\frac{2}{\sqrt{3}}\langle\epsilon|C| \epsilon\rangle C\left({ }^{1} \mathrm{~T}_{2}\right) K(E) .
\end{aligned}
$$

In the above expressions the coefficients $C\left({ }^{1} \mathrm{E}\right)$ and $C\left({ }^{1} \mathrm{~T}_{2}\right)$ occur because of configuration interaction, and are given by:

$$
C\left({ }^{1} \mathrm{~T}_{2}\right)=\left(\alpha^{2}-\gamma^{2}\right): \quad C\left({ }^{1} \mathrm{E}\right)=\left(P^{2}-R^{2}\right)
$$

The Jahn-Teller interaction introduces reduction factors $q$ and $K(E)$. In fact $q \simeq 0.5$ (see Ham 1972) even when there is anharmonicity in the vibronic interaction as inferred by the stress splitting of the GR1 doublet; (see Stoneham 1975, 1977, Lowther 1975). The states given in equation (1) can also be used to give the following expression for the matrix element of the Zeeman operator:

$$
\left\langle{ }^{1} \mathrm{~T}_{2} \epsilon\left|L_{\mathrm{Z}}\right|^{1} \mathrm{~T}_{2} \eta\right\rangle=\left\langle\eta\left|L_{\mathrm{Z}}\right| \epsilon\right\rangle\left[1-2 \beta^{2}\right] K\left(T_{1}\right)
$$

$K\left(T_{1}\right)$ is a quenching factor and, for $T-t$ coupling, $K\left(T_{1}\right) \simeq K(E) \simeq \exp \left(-9 E_{\mathrm{JT}} / \hbar \omega\right)$ (see Ham 1972).

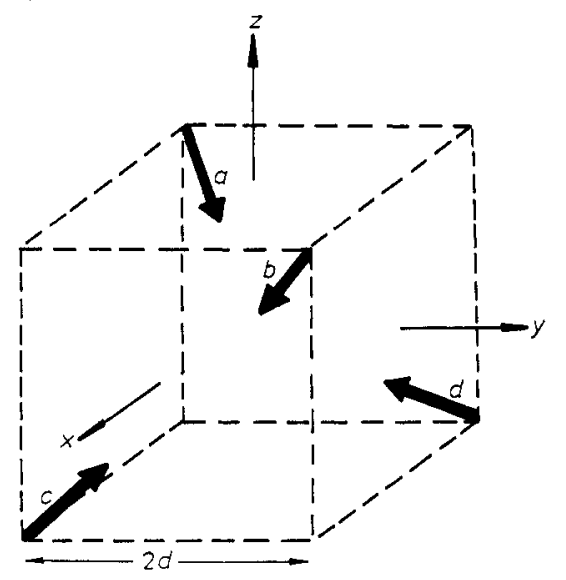

Figure 1. The labelling of the sites and cube axes of the cluster is shown. 


\section{Calculation of the g-factor}

We begin by calculating the g-factor without a Jahn-Teller correction. This corresponds to the original Coulson-Kearsley model. The Jahn-Teller and various other corrections can then be added.

The one-electron orbitals $s$ and $t(\equiv \xi, \eta, \zeta)$ in equations (1) and (2) can be expressed in terms of the dangling bonds shown in figure (1). The (un-normalised) hybrids can be expressed in terms of $\mathrm{p}$ orbitals along the cube axes centred on the appropriate sites:

$$
\begin{aligned}
a & =\left(s_{\mathrm{A}}+x_{\mathrm{A}}+y_{\mathrm{A}}-z_{\mathrm{A}}\right) \\
b & =\left(s_{\mathrm{B}}-x_{\mathrm{B}}-y_{\mathrm{B}}-z_{\mathrm{B}}\right) \\
c & =\left(s_{\mathrm{C}}-x_{\mathrm{C}}+y_{\mathrm{C}}+z_{\mathrm{C}}\right) \\
d & =\left(s_{\mathrm{D}}+x_{\mathrm{D}}-y_{\mathrm{D}}+z_{\mathrm{D}}\right) .
\end{aligned}
$$

The orbital angular momentum operator in (5) regards the centre of the vacancy as origin. However, a local origin on an atomic neighbour simplifies molecular integrals. Transferring the angular momentum operator to appropriate carbon atoms we obtain the following expression for the one-electron matrix element in (5):

$$
\begin{aligned}
\left\langle\eta\left|L_{\mathrm{Z}}\right| \epsilon\right\rangle=N_{t}^{2} & \left\{\left[-\left\langle s_{\mathrm{A}}|\mathrm{d} / \mathrm{d} x| x_{\mathrm{B}}\right\rangle+\left\langle s_{\mathrm{A}}|\mathrm{d} / \mathrm{d} y| y_{\mathrm{B}}\right\rangle+\left\langle x_{\mathrm{A}}|\mathrm{d} / \mathrm{d} x| s_{\mathrm{B}}\right\rangle+\left\langle y_{\mathrm{A}}|\mathrm{d} / \mathrm{d} y| s_{\mathrm{B}}\right\rangle\right] 8 d\right. \\
+ & \left.4\left(S_{\mathrm{AB}}^{P a P \pi}+3 S_{\mathrm{AB}}^{P \pi P \pi}\right)\right\} .
\end{aligned}
$$

Here $N_{t}$ is the normalisation factor. The matrix elements are two-centre integrals and are to be evaluated for two dangling bonds, centred on $a$ and $b$, separated by a distance $\sqrt{ } 8 d$. Numerical values using both Slater and Clementi wavefunctions are shown in table 1. It is interesting to note that the terms $\left\langle s_{A}|\mathrm{~d} / \mathrm{d} x| x_{B}\right\rangle$ etc. are of the same order of

Table 1. Contributions to the orbital moment ( $a=1.684 \mathrm{au}$ ). $N_{t}$ is the normalisation factor associated with the overlaps among the atomic orbitals.

\begin{tabular}{lll}
\hline Contributions to (7) & Slater orbitals & Clement orbitals \\
\hline$\left\langle s_{\mathrm{A}}|\mathrm{d} / \mathrm{d} x| x_{\mathrm{B}}\right\rangle$ & $-0.753 \times 10^{-1}$ & $-0.788 \times 10^{-1}$ \\
$\left\langle s_{\mathrm{A}} \mathrm{d} / \mathrm{d} y \mid y_{\mathrm{B}}\right\rangle$ & $+0.532 \times 10^{-1}$ & +0.1043 \\
$\left\langle x_{\mathrm{A}}|\mathrm{d} / \mathrm{d} x| S_{\mathrm{B}}\right\rangle$ & $-0.906 \times 10^{-1}$ & -0.1374 \\
$\left\langle y_{\mathrm{A}}|\mathrm{d} / \mathrm{d} y| s_{\mathrm{B}}\right\rangle$ & $-0.282 \times 10^{-1}$ & $-0.633 \times 10^{-1}$ \\
$S_{\mathrm{AB}}^{\text {AP }}$ & +0.1219 & +0.1945 \\
$S_{\mathrm{AB}}^{P A \pi}$ & $+0.277 \times 10^{-1}$ & $+0.808 \times 10^{-1}$ \\
$N_{t}$ & 0.4675 & 0.4532 \\
\hline$\left\langle\eta\left|L_{\mathbf{Z}}\right| \epsilon\right\rangle$ & +0.207 & +0.3105 \\
\hline
\end{tabular}

magnitude as the overlap integrals, but that these depend upon whether Slater or Clementi wavefunctions are used. The value of the electronic component of the $g$ factor given by equation (7) is around 0.25 , and is relatively insensitive to the choice of basis functions. This results from a cancellation between many of the terms appearing within the square brackets of equation (7).

The Jahn-Teller reduction factors can be calculated in two independent ways based 
on different assumptions. The first way follows Coulson and Kearsley in taking molecular orbitals built solely from the dangling bonds. From equation (3), taking $q=0 \cdot 5$, we have

$$
B / D=2 K(E) C\left({ }^{1} \mathrm{~T}_{2}\right) / C\left({ }^{1} \mathrm{E}\right)
$$

Since $K\left(T_{1}\right)$ and $K(E)$ are roughly equal for $T-t$ coupling, we can obtain $K\left(T_{1}\right)$ from (8) and the Davies-Penchina values for thestress parameters $\left(B=-0.073 \times 10^{-8} \mathrm{meV} \mathrm{Pa}^{-1}\right.$ and $\left.D=-0.238 \times 10^{-8} \mathrm{MeV} \mathrm{Pa}^{-1}\right)$. We find $K\left(T_{1}\right) \simeq 0.153$. A second method is to make an assumption about the local lattice elastic constants. The Davies-Penchina data and the analysis of Stoneham (1977) can then be combined to give an independent estimate. The assumption made follows the prediction of Lidiard and Stoneham (1967) that the elastic constant for $t$ modes is half that for $e$ modes. This leads to a value of the reduction factor $K\left(T_{1}\right)$ of 0.155 , surprisingly close to the other prediction.

The third factor we must consider is configuration admixture, which gives the $\left(1-2 \beta^{2}\right)$ term in (5). This has been given by Lowther (1975), and lies between 0.93 and $0 \cdot 97$. Collecting the three factors together (i.e. the electronic, Jahn-Teller and configuration interaction components) we find the following results:

\begin{tabular}{|c|c|c|}
\hline Slater (case 1) & $g_{\mathrm{L}}=2.82 \times 10^{-2}$ & $g_{\mathrm{L}}=1.68 \times 10^{-2}$ \\
\hline Slater (case 2) & $g_{\mathrm{L}}=2.23 \times 10^{-2}$ & from experiment \\
\hline Clement & $g_{\mathrm{L}}=4.31 \times 10^{-2}$ & (Douglas and \\
\hline Clementi (case 2) & $g_{\mathrm{L}}=2.84 \times 10^{-2}$ & Runciman 1977) \\
\hline
\end{tabular}

Here cases 1 and 2 correspond to the cases in Lowther (1975).

The predicted values are very close to observation. It is worth noting that two further corrections have not been included. First, all the molecular integrals have been calculated for the geometry of the perfect lattice. The atoms are moved from their sites by both the dynamic Jahn-Teller displacements and by a totally-symmetric distortion. Whilst one can estimate the Jahn-Teller distortion from observed data, the symmetric contribution cannot be obtained without special assumptions. Thus we have been content to make restricted calculations only; these suggest that $g_{\mathbf{L}}$ will not be changed by more than 20 $30 \%$ by any plausible distortion. Secondly, we have followed Coulson and Kearsley in concentrating on the dangling-bond orbitals. If the proper one-electron orbitals extend much further from the vacancy, $g_{\mathbf{L}}$ will be altered. Neither the sign nor the magnitude of the change is easy to estimate, especially given the cancellations which occur between various contributions.

\section{Conclusion}

We have shown that standard molecular-orbital methods can be used to evaluate the $g$ factor of the excited state of the GR1 centre in diamond. The results provide further evidence of the applicability of the Coulson-Kearsley model when generalised to include the significant dynamic Jahn-Teller quenching effects. Both the theory and the DouglasRunciman results suggest that the GR 1 centre is the neutral vacancy, and that the principal transition is from a ${ }^{1} \mathrm{E}$ ground state to a ${ }^{1} \mathrm{~T}_{2}$ excited state.

\section{Acknowledgments}

One of us (AMS) would like to thank the Solid State Physics Research Unit of the Uni- 
versity of the Witwatersrand for its hospitality during the period in which this work was done.

\section{References}

Clark C D and Walker J 1973 Proc. $R$. Soc. A 334 241-57

Coulson C A and Kearsley M J 1957 Proc. R. Soc. A $241433-54$

Coulson C A and Larkins F P 1971 J. Phys. Chem. Solids 32 2245-57

Davies G and Penchina C M 1974 Proc. R. Soc. A 338 359-74

Douglas I N and Runciman W A 1977 J. Phys. C: Solid St. Phys. $102253-9$

Ham F S 1972 Electron Paramagnetic Resonance ed S Geschwind (New York: Plenum) pp 1-119

Lannoo M and Stoneham A M 1968 J. Phys. Chem. Solids 29 1987-2000

Larkins F P and Stoneham A M 1971 J. Phys. C. Solid St. Phys. 4 143-53

Lidiard A B and Stoneham A M 1967 Science and Technology of Industrial Diamonds $11-15$

Lowther J E 1975 J. Phys. C: Solid St. Phys. 8 3448-54 1976 Solid St. Commun. $20933-5$

Stoneham A M 1975 Theory of Defects in Solids (Oxford: Clarendon) ch 27

Stoneham A M 1977 Solid St. Commun. $21339-41$

Yamaguchi T 1962 J. Phys. Soc. Japan 1359-84 